\title{
Deceleration in the Expansion of SN 1993J
}

J. M. Marcaide et al. ${ }^{1}$

Departamento de Astronomia, Universitat de València, E-46100 Burjassot, Spain.

Abstract. With new $6 \mathrm{~cm}$ observations we confirm the self-similar expansion of SN 1993J previously discovered at $3.6 \mathrm{~cm}$ and estimate the expansion deceleration parameter. The results are inconsistent with the existence of a constant pre-explosion stellar wind but otherwise confirm the standard radio supernova model. The first map at $13 \mathrm{~cm}$ showing shell structure is also presented.

The radio structure of SN 1993J in M81 is shell-like and its expansion self-similar (Marcaide et al. 1995a, 1995b). The standard circumstellar interaction model for radio supernovae (Chevalier 1996 and references therein) suggests that the radio emission arises from a shocked region between the supernova ejecta and the circumstellar material (CSM) resulting from the wind of the SN's progenitor star. This model considers supernova ejecta with steep density profiles $\left(\rho_{e j} \propto\right.$ $r^{-n}$ ) shocked by a reverse shock moving inwards from the contact surface and a CSM with density profile $\rho_{c s m} \propto r^{-s}$ shocked by a forward shock moving outwards from the contact surface. For $n>5$, self-similar solutions are possible and the supernova size grows with the power law $t^{m}(t$, time after explosion, and $m=(n-3) /(n-s))$.

We present in Figure 1.a a preliminary map at $13 \mathrm{~cm}$ from 1 October 1995 which shows, for the first time at this wavelength, the shell structure of SN 1993J. In Figure 1.b we plot the results of the expansion at $6 \mathrm{~cm}$ through October 1996 (42 months after explosion); the corresponding $6 \mathrm{~cm}$ maps are presented by Marcaide et al. (1997). The result at $13 \mathrm{~cm}$ has been included in Figure 1.b. A fit of a function of the form $\mathrm{R} \propto t^{m}$ to the data gives an $m=0.89 \pm 0.04$. (Marcaide et al. (1997) obtain $m=0.86 \pm 0.02$ with 3.6 and $6 \mathrm{~cm}$ data.) Within the framework of self-similar models, the opacity follows $\tau \propto t^{2 m(-s+0.5)}$. Combining the result by Van Dyk et al. (1994) with our estimate of $m=0.89 \pm 0.04$ we obtain $s=1.62_{-0.25}^{+0.14}$. This value is lower than the usually assumed $s=2$ in the standard model for a constant stellar wind but very close to the value $s=1.7$ given by Fransson et al. (1996) to explain X-ray emission. Suzuki \& Nomoto (1995) suggest $s=3$ for the region swept by the supernova between months 24 and 42 after explosion. Our results argue against their model.

The self-similar case with $m=0.89$ and $s=1.62$ gives an ejecta density profile of $n=14.2_{-4.2}^{+10.5}$. In the standard model, for values $n=14.2$ and $s=1.62$, the forward shock radius is $\sim 20 \%$ larger than the radius of the contact surface

${ }^{1}$ J.M. Marcaide ${ }^{1}$, A. Alberdi ${ }^{2,3}$, E. Ros ${ }^{1}$, P. Diamond ${ }^{4}$, I. I. Shapiro ${ }^{5}$, J. C. Guirado ${ }^{1}$, D. L. Jones ${ }^{6}$, F. Mantovani ${ }^{7}$, M. A. Pérez-Torres ${ }^{1}$, R. A. Preston ${ }^{6}$, R. T. Schilizzi ${ }^{8,9}$, R. A. Sramek ${ }^{4}$, C. Trigilio ${ }^{10}$, S.D. Van Dyk ${ }^{11}, \mathrm{~K}$. W. Weiler ${ }^{12}$, A. R. Whitney ${ }^{13}-{ }^{1}$ Universitat de València, Burjassot 46100, Spain; ${ }^{2}$ LAEFF-INTA, 28080 Madrid, Spain; ${ }^{3}$ IAA-CSIC, 18080 Granada, Spain; ${ }^{4}$ NRAO, Socorro, NM 87801, USA; ${ }^{5}$ Harvard-Smithsonian CfA, Cambridge, MA 02138, USA; ${ }^{6} \mathrm{JPL}$, Pasadena, CA 91109, USA; ${ }^{7}$ IRA-CNR, Bologna 40129, Italy; ${ }^{8} \mathrm{JIVE}$, 7990 AA Dwingeloo, Netherlands; ${ }^{9}$ Leiden Observatory, 2300 RA Leiden, Netherlands; ${ }^{10}$ IR.ACNR, Noto, Italy; ${ }^{11}$ UCLA, Los Angeles, CA 90095; ${ }^{12}$ NRL, Washington, DC 20375-5320; ${ }^{13}$ MIT-Haystack Observatory, Westford, MA 01886, USA 
SN 1993J - Observations
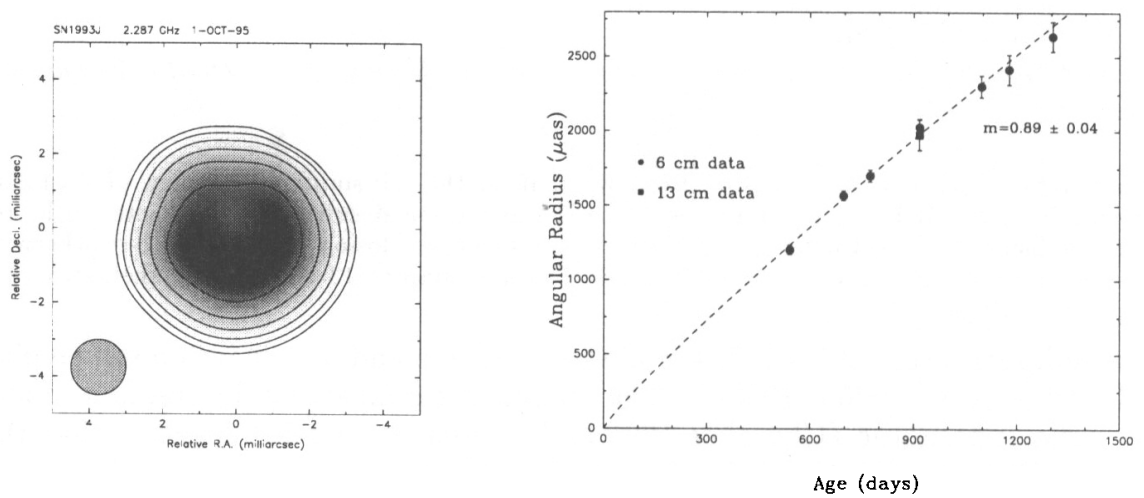

Figure 1. a) Map at $13 \mathrm{~cm}$ of SN 1993J from 1 October 1995, 907 days after explosion, showing the shell structure for the first time at that wavelength. The maximum brightness is $12.7 \mathrm{mJy} /$ beam. The circular gaussian beam used in the convolution to obtain this CLEAN map is shown in the lower left and has FWHM of 1.5 mas; b) Angular radius of SN 1993J vs. days after explosion. The line is a fit of a function of the type $\mathrm{R} \propto t^{m}$ (t, time after explosion) to $6 \mathrm{~cm}$ data. The value of $m$ which corresponds to such a fit is $0.89 \pm 0.04$. Combining these data (not including the $13 \mathrm{~cm}$ result) with the $3.6 \mathrm{~cm}$ data, Marcaide et al. (1997) obtain $m=0.86 \pm 0.02$.

between shocked supernova ejecta and shocked CSM. In contrast, Marcaide et al. (1995b) estimate that the width of the radio shell is about $40 \%$ the size of the inner radius; the present results confirm those findings. Perhaps clumpy ejecta and/or CSM can broaden the shell (Houck \& Fransson 1996), thus enabling the standard model and the observational results to be reconciled. If the physical picture of the radio and $\mathrm{H} \alpha$ emission in the standard model were correct, there should also be a correlation between the slowdown in the growth rate we measure and a decrease of the maximum speed measurable in the $\mathrm{H} \alpha$ emission which originates in the neighborhood of the inner radius. Between months 12 and 42 the expansion speed as measured by VLBI has decreased by $\sim 15 \%$. This decrease should also be observable in the $\mathrm{H} \alpha$ emission.

\section{References}

Chevalier, R. A. 1996. in Radio Emission from the Stars and the Sun, eds. A. R. Taylor, \& J. M. Paredes (San Francisco: ASP Conference Series, Vol. 93), 125-133.

Fransson, C., Lundqvist, P., Chevalier, R. A., 1996. ApJ, 461, 993-1008.

Houck, J. C. \& Fransson, C., 1996. ApJ, 456, 811-832.

Marcaide, J. M., et al. 1995a. Nature, 373, 44-45.

Marcaide, J. M., et al. 1995b, Science, 270, 1475-1478.

Marcaide, J. M., et al. 1997. ApJ, 486, L31-34.

Suzuki, T. \& Nomoto, K., 1995. ApJ, 455, 658-669.

Van Dyk, S. D., et al. 1994. ApJ, 432, L115-118. 